\title{
The relation between Helicobacter pylori and gastroesophageal reflux disease in KSA
}

\author{
Faisal Alziyadi ${ }^{1}$, Nouf Alshammeri ${ }^{2}$, Ali Alamer ${ }^{3}$, Fatimah Altarouti ${ }^{4}$, Nesreen Aljehani ${ }^{5}$, \\ Khalid Asiri $^{6}$, Fardus Almarghalani ${ }^{7}$, Easa Albalwi ${ }^{8}$, Malik Almohideb ${ }^{9}$, Ameen hayyan ${ }^{10}$
}

\author{
${ }^{1}$ Klakh Primary Health Care Center, Taif, KSA \\ ${ }^{2}$ King Khalid Hospital, Hail, KSA \\ ${ }^{3}$ Prince Saud Bin Jalawy Hospital, Alahsa, KSA \\ ${ }^{4}$ King Khalid University, Abha, KSA \\ ${ }^{5}$ Ibn Sina National College, Jeddah, KSA \\ ${ }^{6}$ Muhayl General Hospital, Muhayl, KSA \\ ${ }^{7}$ King Fahad General Hospital, Jeddah, KSA \\ ${ }^{8}$ King Saud Bin Abdulaziz University for Health Sciences, Jeddah, KSA \\ ${ }^{9}$ Imam Muhammad Ibn Saud Islamic University, Riyadh, KSA \\ ${ }^{10}$ Safwa General Hospital, Dammam, KSA
}

Received: 12 July 2018

Revised: 08 August 2018

Accepted: 09 August 2018

\section{*Correspondence:}

Dr. Faisal Alziyadi,

E-mail: Dr.alotiby@hotmail.com

Copyright: (c) the author(s), publisher and licensee Medip Academy. This is an open-access article distributed under the terms of the Creative Commons Attribution Non-Commercial License, which permits unrestricted non-commercial use, distribution, and reproduction in any medium, provided the original work is properly cited.

\begin{abstract}
Estimates say that Helicobacter pylorus affects more than half of the general population over the world. Its prevalence varies significantly among different areas. Studies done in 1990 reported a prevalence of $70 \%$ of people older than 20 years who live in Saudi Arabia, and 40\% of people aged 5-10 years. The first line regimen of helicobacter pylori infections in Saudi Arabia is 7 days of triple therapy. However, in areas of high antibiotic resistance, or in cases of failure of triple therapy, a 'rescue' quadruple therapy (that contains bismuth) is indicated. In this study we aimed to look at the prevalence of $H$. pylori infection and its association with gastroesphageal reflux disease in Saudi Arabia. We also tried to understand the approach for diagnoses and treatment in the Kingdom. We did a systematic search for helicobacter pylori and GERD in Saudi Arabia using PubMed search engine (http://www.ncbi.nlm.nih.gov/) and Google Scholar search engine (https://scholar.google.com). The following search terms were used: H. pylori, gastroesophageal reflux disease, prevalence of GERD in Saudi Arabia, lifestyle Saudi Arabia, gastroenterology Saudi Arabia. All studies done shows prevalence in Saudi Arabia to be relatively high (reaching 70\%) and increasing. Despite being highly prevalent, no solid guidelines are present to help clinicians diagnose and treat helicobacter pylori infection properly. These, along with other causes like improper use of antibiotics have led to the emerging of new strains that are resistant to typical antibiotic treatment.
\end{abstract}

Keywords: Helicobacter pylori infection, Lifestyle Saudi Arabia, Prevalence of GERD in Saudi Arabia, Antibiotic use in Saudi Arabia

\section{INTRODUCTION}

Helicobacter pylori bacteria is an opportunistic organism that is gram-negative and spiral-shaped. It is estimated that it effects more than half of the general population over the world. ${ }^{1}$ Exact rates of infection vary significantly depending on geographical distribution, hygiene, social and economic levels, age, ethnic groups, and other 
factors. Long term infection with helicobacter pylori bacteria has been found to be associated with high risk of developing significant diseases. ${ }^{2}$ These include, gastric cancers (that are found in up to $1 \%$ patient infected with helicobacter pylori), gastroesophageal reflux disease (GERD), peptic ulcers, and other diseases. ${ }^{3-6}$ On the other hand, although helicobacter pylori infection is relatively highly prevalent, most infected people are asymptomatic. A possible explanation for this is the genetic differences between several strains of the bacteria itself. ${ }^{7,8}$ Also, the presence of different virulence factors can largely affect clinical picture and pathogenicity. These virulence factors include urease (UreA), outer membrane protein (OMP), vacuolating toxin (vacA), and cytotoxin associated gene A (cagA). ${ }^{9-12}$ Several reports have suggested the presence of a positive correlation between cagA and vacA genotypes, and disease severity. ${ }^{13,14}$

Because of this clinical variation of helicobacter pylori strains, and their associated clinical presentations, most helicobacter pylori cases are treated with protocols that include multidrugs (triple or quadruple therapy). ${ }^{8}$ The aim behind this is the treatment and prevention of peptic ulcer disease, and the reduction of gastric cancer risk. Triple therapy protocols include 2 antibiotics (like amoxicillin and clarithromycin) plus a proton pump inhibitor (PPI). ${ }^{15-17}$ When the prevalence of antibiotics resistance is higher than $20 \%$, using quadruple therapy is recommended. In the case of quadruple therapy, we use metronidazole, a tetracycline, along with bismuth citrate and a PPI. ${ }^{16}$ However, there are still cases where treatment failure occur. Reasons behind this are numerous and include the presence of resistant strains of the organism, infection recurrence, and antibiotics abuse. ${ }^{19-22}$ All these previously mentioned problems have made helicobacter pylori infection an important issue in Saudi Arabia, with significant burden on Saudi society.

\section{METHODS}

We did a systematic search for helicobacter pylori and GERD in Saudi Arabia using PubMed search engine (http://www.ncbi.nlm.nih.gov/) and Google Scholar search engine (https://scholar.google.com). Our search also looked for the prevalence of helicobacter Pylori infection in Saudi Arabia, and the presence of treatment resistance in the Kingdom. All relevant studies were retrieved and discussed. We only included full articles. Terms used for researching were: Helicobacter Pylori, gastroesophageal reflux disease, prevalence of GERD in Saudi Arabia, lifestyle Saudi Arabia, gastroenterology Saudi Arabia

\section{Data extraction}

Two reviewers have independently reviewed the studies, abstracted data and disagreements were resolved by consensus. Studies were evaluated for quality and a review protocol was followed throughout.

\section{PREVALENCE AND TREATMENT OF HELICOBACTER PYLORI INFECTIONS IN SAUDI ARABIA}

Prevalence of $H$. pylori varies significantly among different areas. In general, developed countries show a significantly lower prevalence of infection when compared to developing countries, in which prevalence can be as high as $80 \%$. Factors that mainly affect this prevalence include age, sex, genetic predisposition, racial and ethnic disparities, geographical distribution, hygiene, and other risk factors. ${ }^{23}$

The kingdom of Saudi Arabia is considered to be a developing country where people of several ethnicities and socioeconomic status live. Many reports have described the prevalence of $H$. pylori infection in the Kingdom. A study published my Moagel et al in 1990 has reported a prevalence of $70 \%$ of people older than 20 years who live in Saudi Arabia, and $40 \%$ of people aged 5-10 years. These numbers are considered to be very high making Saudi Arabia one of the most prevalent countries of $H$. pylori infection around the world. ${ }^{24}$ Marie et al, also published a study in 2008 that found that $67 \%$ of people who live in Saudi Arabia were seropositive. They also found that this prevalence increased significantly with age and among females. Another study was conducted in Makkah city and found that the prevalence of helicobacter pylori infection was as high as $61 \%$ Salih et al, published a study in 2009 and they found that about $33 \%$ of children younger than 10 years were seropositive for $H$. pylori. ${ }^{3,25,26}$ Another study conducted by Hunt et al, has found that prevalence of $H$. pylori among adults in Saudi Arabia has has been increasing and reached $80 \%{ }^{21}$ Saudi Arabia has a population of about 27 million people, of which 8.4 million are foreigners ${ }^{26}$. Most previously mentioned studies were limited by selection bias, small samples, and possible lack of generalizability.

Similar to other places in the world, H. pylori infections are treated mainly with either triple or quadruple therapy in Saudi Arabia. However, no drugs regimen has been found to achieve complete eradication. Reasons behind this possibly include noncompliance of patients, improper use of antibiotics, genetic predisposition, the presence of several $H$. pylori strains, and the variation of bioavailability of drugs in the lumen. ${ }^{19}$ Generally, the first line regimen of $H$. pylori infections in Saudi Arabia is a week of triple therapy. ${ }^{17}$ However, in areas of high antibiotic resistance, or in cases of failure of triple therapy, a 'rescue' quadruple therapy (that contains bismuth) is indicated. ${ }^{22}$

The prevalence of antibiotics resistance has also been increasing in Saudi Arabia, making the use of classic first-line therapy not beneficial in many cases. The most important factor in this is the improper use of antibiotics in clinical settings, and antibiotics abuse by patients. ${ }^{27}$ To decrease rates of resistance against $H$. pylori regimens, it 
is essential to use combinations of several antibiotics as a first line choice in Saudi Arabia. ${ }^{18}$

Resistant strains of $H$. pylori have been emerging around the world, and this includes Saudi Arabia. This increase in treatment resistance has necessitated the continuous improvement of treatment regimens that are used against H. pylori. A previous study has found that while resistance was present in about $35 \%$ of patients in Saudi Arabia in 1988, this significantly increased and reached $79.5 \%$ in 1996. It was also found that female had significantly higher levels of resistance than males. Despite this dramatic increase between 1988 and 1996, prevalence of $H$. pylori treatment resistance in Saudi Arabia stabilized in the following period, and was found to be $69.5 \%$ in $2008 .^{28}$ Prevalence of $H$. pylori treatment resistance in Saudi Arabia was found to vary between cities. Western Saudi Arabia, for example, has a prevalence of resistance that is about $48 \%$. $^{29}$

\section{DIAGNOSIS OF HELICOBACTER PYLORI INFECTIONS IN SAUDI ARABIA}

When it comes to diagnosing $H$. pylori infections in Saudi Arabia, the Saudi Medical Council has not released any guidelines regarding this issue. Therefore, Saudi clinicians usually use guidelines from Europe or the United States. Urea breath test (UBT) and stool antigen test are the main methods used for the diagnosis of $H$. pylori among adults. Another method is serology, which is, however, limited by its inability to differentiate active and past disease. It can still be used in patients who do not have a prior history of $H$. pylori. In cases of relapse, endoscope with culture and antibiotics sensitivity tests are done. PCR is considered an alternative for cultures. Testing for $H$. pylori eradication is usually performed 4-8 weeks following treatment regimens. ${ }^{17,30}$ In general, guidelines that are based on Saudi patients and Saudi epidemiology should be formed for the diagnosis of $H$. pylori.

\section{HELICOBACTER PYLORI INFECTION AND ASSOCIATED GERD}

Infection with $H$. pylori is known to be an important cause of several gastrointestinal diseases. These diseases include chronic gastritis, gastric atrophy, intestinal metaplasia (all of which are associated with the later development of gastric cancer), peptic ulcer, and gastroesophegeal reflux disease (GERD). However, the association between $H$. pylori infection, and gastroesophegeal reflux disease (GERD) is still controversial. ${ }^{31}$ This is due to lack of definitive evidence that is with or against this association. Moreover, several studies have found that $H$. pylori infections negatively correlate with gastroesophegeal reflux disease. However, these studies still showed significant decline of clinical picture in gastroesophegeal reflux disease patients who are infected with $H$. pylori. A previous study that was conducted in Turkey did not find any significant association between $H$. pylori infection and gastroesophegeal reflux disease. ${ }^{32}$ On the other hand, a study that was conducted in Sardinia among 5,156 patients using endoscopy, has concluded that $H$. pylori infection is significantly higher in patients with gastroesophegeal reflux disease (odds ratio was 1.17, with a $\mathrm{p}$ value of $0.04 .^{33} \mathrm{In}$ addition, the pathogenicity, in which $H$. pylori infection will affect the gastrointestinal tract and become involved in gastroesophageal reflux disease is still controversial and not well understood. A main reason for this lack of knowledge is the major disparities among regions and epidemiological groups, and the presence of many genetic types and strains of the pathogen. $H$. pylori bacteria continues to be one of the most important causes in the development and recurrence of peptic ulcer (although non-steroidal anti-inflammatory drugs are becoming of extreme importance recently).

\section{HELICOBACTER PYLORI INFECTION AND ASSOCIATED BARRET ESOPHAGUS}

Barret esophagus, on the other hand, in a large study on several racial and ethnic groups in the United States, was found to negatively correlate with $H$. pylori infection. This study, conducted by Sonnenberg et al, was a case control study that was performed on 596,479 patients, of which $12.8 \%$ (76,475 patients) had a Barret esophagus that is histologically confirmed. Both increasing age and male sex were found to have the strongest association with the development of Barret esophagus. ${ }^{34}$ Another report discussed this issue and the possible relationship between $H$. pylori infection, and Barret esophagus, and esophageal adenocarcinoma. In this study, authors have concluded that $H$. pylori infection is associated with lower incidence of Barret esophagus. However, they still recommended to perform other larger cohort studies where all possible confounding factors are controlled. ${ }^{35}$

\section{EFFECTS OF ERADICATION OF HELICOBATER PYLORI INFECTION ON GASTROESOPHAGEAL REFLUX DISEASE}

Gastric atrophy has been found to reverse upon eradication of $H$. pylori bacteria from the gastrointestinal tract of infected individuals. However, intestinal metaplasia was not found to be reversible even after complete eradication of the pathogen. Therefore, scientists and researchers have been largely interested in the pathophysiological mechanisms in which chronic gastritis progresses to atrophy and metaplasia. The role of H. pylori in all these sequences has also been studied well to identify possible ways of preventing atrophy and metaplasia. Moreover, the role of proton pump inhibitors (which are a part of $H$. pylori treatment regimens) in the development and progression of atrophy and metaplasia has also been studied well. ${ }^{36}$

Previous reports claimed that eradication of $H$. pylori might cause negative changes in the production of gastric acid, and these changes would lead to worsened 
gastroesophageal reflux disease. However, several studies could not prove this, and thus the eradication of $H$. pylori infection continues to be recommended. ${ }^{37}$

A previous study that was performed in Taiwan on 325 patients who were diagnosed with gastroesophageal reflux. These patients were divided into two groups according to being $H$. pylori seropositive or seronegative. Patients with positive $H$. pylori infection were further divided into two groups. One of which received eradication therapy, and the other group did not. All these patients were then treated with a proton pump inhibitor (esomeprazole) until becoming asymptomatic. Investigators performed an endoscopy on all patients three times: at the beginning of the study, after 1 year, and after 2 years. Authors found that patients who tested negative for $H$. pylori did not have any worsening of gastric atrophy or metaplasia. Patients who tested positive for $H$. pylori and received treatment until eradication were found to have improved metaplasia and atrophy when they were followed. On the other hand, patients who had an untreated $H$. pylori infection were found to have increasing atrophy and metaplasia when they were followed up later. Results of this study provide an important evidence that favors eradication of $H$. pylori infection in patients with gastroesophageal reflux disease. $^{38}$

Another clinical trial on patients with gastroesophageal reflux disease was conducted. In this study, 155 patients were either started on a proton pump inhibitor (omeprazole) or underwent surgery. The omeprazole group contained 40 patients who had a confirmed $H$. pylori infection, and the surgery group contained 53 patients who had confirmed $H$. pylori infection. The study continued for three years, and at the end of the study, no significant difference between all groups was noted. The main conclusion of this study was that the use of proton pump inhibitors did not increase the risk of developing gastric atrophy and intestinal metaplasia. Another study was later performed for a longer period, but on a smaller sample reached similar conclusions. However, both studies are still limited by small sample size, and possible selection bias. ${ }^{39}$

A larger similar clinical trial was later conducted to assess the effects of proton pump inhibitors treatment. This study included 180 patients in the proton pump inhibitor group, and 158 patients in the surgery group. Only about $13 \%$ of all included patients had a $H$. pylori infection. Results of this trial showed that none of the patients in both groups developed gastric atrophy or intestinal metaplasia. A subgroup analysis was conducted on patients with $H$. pylori infection found that these patients had progression of gastric atrophy and metaplasia. However, caution should be present when interpreting these results due to the very small number of patient who tested positive for $H$. pylori. ${ }^{40}$

\section{CONCLUSION}

H. pylori infection is considered an important health issue both worldwide and in Saudi Arabia. Prevalence of $H$. pylori infection varies among countries, ethnic and racial groups, and different socioeconomic levels. Other factors that affect the prevalence of $H$. pylori include age, sex, hygiene, and genetic predisposition. Several studies were conducted on Saudi populations to calculate the prevalence of $H$. pylori infections, and these studies have reached several conclusions. However, in all these studies, prevalence was found to be relatively high (reaching 70\%) and increasing. Despite being highly prevalent in Saudi Arabia, no solid guidelines are present to help clinicians diagnose and treat $H$. pylori infection properly. This, along with other causes like improper use of antibiotics have led to the emerging of new strains that are resistant to typical antibiotic treatment. $H$. pylori infection has been suggested to be associated with gastroesophageal reflux disease. However, this association has not been proven until now, and this issue remains to be controversial. Despite inconsistent evidence in the association between $H$. pylori infection and gastroesophageal reflux disease, patients with gastroesophageal reflux disease are recommended to undergo screening for $H$. pylori infection, and those who test positive are recommended to undergo eradication therapy. This therapy aims at the reduction future sequelae that may be caused from Helicobacter pylori.

Funding: No funding sources

Conflict of interest: None declared

Ethical approval: Not required

\section{REFERENCES}

1. Dunn BE, Cohen H, Blaser MJ. Helicobacter pylori. Clin Microbiol Rev. 1997;10:720-41.

2. Blaser MJ. Epidemiology and pathophysiology of campylobacter pylori infections. Rev Infect Dis. 1990;12(1):99- 106.

3. Salih BA. Helicobacter pylori infection in developing countries: The burden for how long. Saudi J Gastroenterol. 2009;15:201-7.

4. Khudor MH, Strak SK, Issa AH. Isolation of CagA and VacA genes from $\mathrm{H}$. pylori infected patients with various gastroduodenal lesions. Bas J Surg. 2010;16:31-8.

5. Fact sheet on Helicobacter pylori and Cancer. 2013. Available at: https://www.cancer.gov/aboutcancer/causes-prevention/risk/infectious-agents/hpylori-fact-sheet. Accessed on 3 July 2018.

6. Ernst PB, Peura DA, Crowe SE. The translation of Helicobacter pylori basic research to patient care. Gastroenterology. 2006;130:188-206.

7. Björkholm B, Sjolund BM, Falk PG, Berg OG, Engstrand L, Andersson DI. Mutation frequency and biological cost of antibiotic resistance in Helicobacter pylori. Proc Natl Acad Sci USA. 2001;98:14607-12. 
8. Falush D, Kraft C, Taylor NS, Correa P, Fox JG, Achtman $\mathrm{M}$, et al. Recombination and mutation during long-term gastric colonization by Helicobacter pylori: Estimates of clock rates, recombination size, and minimal age. Proc Natl Acad Sci USA. 2001;98:15056-61.

9. Alvi A, Ansari SA, Ehtesham NZ, Rizwan M, Devi $\mathrm{S}$, Sechi LA, et al. Concurrent proinflammatory and apoptotic activity of a Helicobacter pylori Protein (HP986) points to its role in chronic persistence. PLoS One 2011;6:e22530.

10. Rizwan M, Alvi A, Ahmed N. Novel Protein Antigen (JHP940) from the Genomic Plasticity Region of Helicobacter pylori Induces tumor necrosis factor alpha and interleukin- 8 secretion by human macrophages. J Bacteriol. 2008;190:114651.

11. Kusters JG, van Vliet AH, Kuipers EJ. Pathogenesis of Helicobacter pylori infection. Clin Microbiol Rev. 2006;19:449-90.

12. Axon AT. Are all helicobacter equal? Mechanisms of gastroduodenal pathology and their clinical implications. Gut 1999;45(1):11-4.

13. Jenks PJ, Megraud F, Labigne A. Clinical outcome after infection with Helicobacter pylori does not appear to be reliably predicted by the presence of any of the genes of the cag pathogenicity island. Gut. 1998;43:752-8.

14. Van der Ende A, Rauws EA, Feller M, Mulder CJ, Tytgat GN, Dankert J. Heterogeneous Helicobacter pylori isolates from members of a family with a history of peptic ulcer disease. Gastroenterol. 1996;111:638-47.

15. Buta N, Tanih NF, Ndip RN. Increasing trend of metronidazole resistance in the treatment of Helicobacter pylori infection: A global challenge. Afr J Biotechnol. 2010;9:1115-21.

16. Megraud F. $H$. pylori antibiotic resistance: Prevalence, importance, and advances in testing. Gut. 2004;53:1374-84.

17. Chey WD, Wong BC:Practice Parameters Committee of the American College of Gastroenterology. Guideline on the Management of Helicobacter pylori infection. Am J Gastroenterol. 2007;102:1808-25.

18. De Francesco V, Ierardi E, Hassan C, Zullo A. Helicobacter pylori therapy: Present and Future. World J Gastrointest Pharmacol Ther. 2012;3:6873.

19. O'connor A, Taneike I, Nami A, Fitzgerald N, Murphy P, Ryan B, et al. Helicobacter pylori resistance to metronidazole and clarithromycin in Ireland. Eur J Gastroenterol Hepatol. 2010;22:11237.

20. $\mathrm{Wu} \mathrm{W}$, Yang $\mathrm{Y}$, Sun G. Recent insights into antibiotic resistance in Helicobacter pylori eradication. Gastroenterol Res Pract. 2012;2012:723183.
21. De Francesco V, Giorgio F, Hassan C, Manes G, Vannella L, Panella C, et al. Worldwide H. pylori Antibiotic Resistance: A Systematic Review. J Gastrointestin Liver Dis. 2010;19:409-14.

22. Megraud F. Antibiotic resistance in Helicobacter pylori infection. Br Med Bull. 1998;54:207-16.

23. Taneike I, Nami A, O Connor A, Fitzgerald N, Murphy P, Qasim A, et al. Analysis of drug resistance and virulence-factor genotype of Irish $\mathrm{H}$ pylori strains:Is there any relationship between resistance to metronidazole and cagA status? Aliment Pharmacol Ther. 2009;30:784-90.

24. Al Moagel MA, Evans DG, Abdulghani ME, Adam E, Evans DJ Jr, Malaty HM, et al. Prevalence of Helicobacter pylori (formerly Campylobacter) Infection in Saudi Arabia, and comparison of those with and without upper gastrointestinal symptoms. Am J Gastroenterol. 1990;85:944-8.

25. Marie MA. Patterns of $H$. pylori Resistance to metronidazole, clarithormycin and Amoxicillin in Saudi Arabia. J Bacteriol Virol. 2008;38:173-8.

26. Khan MA, Ghazi OH. Helicobacter pylori infection in asymptomatic subjects in Makkah. J Pak Med Assoc. 2007;57:114-6.

27. Available at: http://www.saudiembassy.net/about/ country-information/. Accessed on 3 July 2018.

28. Marie MA. Seroprevalence of Helicobacter pylori infection in large series of patients in an urban area of Saudi Arabia. Korean J Gastroenterol. 2008;52:226-9.

29. Momenah AM, Asghar AH. Prevalence and antibiotic resistance among Helicobacter pylori clinical isolates from main Hospitals in the Western Region of Saudi Arabia. Pak J Med Sci. 2008;24:100-3.

30. Lee SY. Current progress toward eradicating Helicobacter pylori in East Asian countries: differences in the 2013 revised guidelines between China, Japan, and South Korea. World J Gastroenterol. 2013;20:1493-502.

31. Kandulski A, Malfertheiner P. Helicobacter pylori and gastroesophageal reflux disease. Curr Opin Gastroenterol. 2014;30:402-7.

32. Bor S, Kitapcioglu G, Kasap E. Prevalence of gastroesophageal reflux disease in a country with a high occurrence of Helicobacter pylori. World J Gastroenterol. 2017;23:525-32.

33. Dore MP, Pes GM, Bassotti G, Farina MA, Marras G, Graham DY. Risk factors for erosive and nonerosive gastroesophageal reflux disease and barrett's esophagus in Nothern Sardinia. Scand J Gastroenterol. 2016;51:1281-7.

34. Sonnenberg A, Turner KO, Spechler SJ, Genta RM. The influence of Helicobacter pylori on the ethnic distribution of barrett's metaplasia. Aliment Pharmacol Ther. 2017;45:283-90.

35. Tomasello G, Giordano F, Mazzola M, Jurjus R, Jurjus A, Damiani P, et al. Helicobacter pylori and 
barretts esophagus: a protective factor or a real cause? J Biol Regul Homeost Agents. 2017;31:9-15.

36. Vakil N. Rationale for a Helicobacter pylori Test and Treatment Strategy in Gastroesophageal Reflux Disease. Gastroenterol Clin N Am. 2015;28:971-85.

37. Malfertheiner P, Megraud F, O'Morain CA, et al. Management of Helicobacter pylori infection-the maastricht V/florence consensus report. Gut. 2017;66:6-30.

38. Yang HB, Sheu BS, Wang ST, Cheng HC, Chang WL, Chen WY. H. pylori eradication prevents the progression of gastric intestinal metaplasia in reflux esophagitis patients using long-term esomeprazole. Am J Gastroenterol. 2009;104(7):1642-9.

39. Lundell L, Miettinen P, Myrvold HE, Pedersen SA, Thor K, Andersson A, et al. Lack of effect of acid suppression therapy on gastric atrophy. Nordic GERD Study Group. Gastroenterology. 1999;117(2):319-26.

40. Fiocca R, Mastracci L, Attwood SE, Ell C, Galmiche JP, Hatlebakk J, et al. LOTUS Trial Collaborators. Gastric exocrine and endocrine cell morphology under prolonged acid inhibition therapy: results of a 5-year follow-up in the LOTUS trial. Aliment Pharmacol Ther. 2012;36(10):959-71.

Cite this article as: Alziyadi F, Alshammeri N, Alamer A, Altarouti F, Aljehani N, Asiri K, et al. The relation between Helicobacter pylori and gastroesophageal reflux disease in KSA. Int $\mathbf{J}$ Community Med Public Health 2018;5:3691-6. 